\title{
Allopatric divergence limits cheating range and alters genetic requirements for a cooperative trait
}

\author{
Kaitlin A. Schaal ${ }^{\star}$, Yuen-Tsu Nicco Yu, Marie Vasse, and Gregory J. Velicer \\ Institute of Integrative Biology, ETH Zürich, 8092 Zürich, Switzerland \\ * Corresponding author. Email: kaitlin.schaal@env.ethz.ch
}

Social and genomic context may constrain the fates of mutations in cooperation genes. While some mechanisms limiting cheaters evolve in the presence of cheating, here we ask whether cheater resistance can evolve latently even in environments where cooperation is not expressed and cheaters are absent. The bacterium Myxococcus xanthus undergoes cooperative multicellular development upon starvation, but developmentally defective cheaters can outcompete cooperators within mixed groups. Using natural isolates and an obligate cheater disrupted at the developmental-signaling gene csgA, we show that cheating range is narrow among natural strains due to antagonisms that do not specifically target cheaters. Further, we mixed the cheater with closely related cooperators that diverged from it allopatrically in nutrient-rich environments in which cooperative development does not occur, showing that even slight divergence under these conditions can eliminate cheating phenotypes. Our results suggest that such cooperation- and cheater-blind divergence can generate a geographic mosaic of local cheater-cooperator compatibility patches that limit cheater spread. We also ask whether genomic divergence can shape the fitness effects of disrupting a cooperation gene. Construction of the same csgA mutation in several natural-isolate cooperators generated a wide range of pure-culture sporulation phenotypes, from a complete defect to no defect. Thus, we find that epistatic interactions limit the range of genomes within which a mutation creates a cooperation defect. Moreover, these results reveal Developmental System Drift in a microbial system because sporulation proficiency is conserved across the natural isolates despite divergence in the role of $\operatorname{csg} A$.

Significance statement. Selection on cooperators exploited by obligate cheaters can induce evolution of resistance to cheating. Here we show that cooperators can also rapidly evolve immunity to cheating simply as a byproduct of evolutionary divergence in environments in which cooperation and cheating at the focal trait do not occur because the trait is not expressed. We also find that differences in the genomic context in which a cooperation-gene mutation arises can profoundly alter its phenotypic effect and determine whether the mutation generates a social defect at all - a pre-requisite for obligate cheating. These findings suggest that general divergence of social populations under a broad range of environmental conditions can restrict both the set of mutations that might generate social defectors in the first place and the host range of such defectors once they arise.

Cooperative systems throughout the tree of life have persisted for long periods due to passive and active mechanisms that limit obligately non-cooperative "cheaters" (see Semantics in Methods). Mechanisms that stabilize cooperative relationships function by promoting social interactions that direct a higher average reproductive benefit to cooperative genotypes than to cheaters (1). Many such mechanisms have been proposed or demonstrated (e.g. 1-15), but their actual relative contributions to limiting and shaping spatial patterns of obligate cheating in natural populations are often unclear.

Cooperators can evolve higher fitness than cheaters in response to different types of selective pressures. This has been demonstrated in several microbial experimental systems in which cooperators evolved to outcompete cheaters by adapting either to abiotic features of the environment in which cooperation and cheating occur or to cheaters themselves $(4,6,12,14$, 16-18). In those studies, the relevant cooperative trait was important to fitness in the environments in which cooperators evolved, and cheaters that exploited the trait were part of those environments. Cooperators might also latently evolve resistance to being cheated on in one environment (e.g. a nutrient-poor habitat) while evolving in other environments in which the exploitable cooperative trait is not expressed (e.g. nutrient-rich habitats) and in allopatry from cheaters a hypothesis we investigate here.

Allopatric divergence often profoundly alters biotic interactions. For example, allopatric speciation is an important form of divergence in animals and plants in 
which barriers to reproduction evolve blindly between spatially separate populations $(19,20)$. In social insects, allopatric divergence affects social-parasitism behaviors $(21,22)$. In microbes, allopatric divergence of social types can increase inter-strain antagonism (23) and also generate kin-discriminatory colony interactions (24), fitness asymmetries specific to social interactions (25), and social exploitation among cooperation-proficient genotypes (25).

The soil bacterium Myxococcus xanthus is a wellstudied microbial cooperative system that displays social behaviors such as swarming motility (26) and multicellular development into spore-filled fruiting bodies (27). Cooperative lab strains of $M$. xanthus which are proficient at development sometimes yield mutants that can cheat on them - that is, the mutants have higher fitness than the cooperative parent in mixed groups due to a mutation which prevents them from producing functional signal molecules necessary for normal development (2). Lab-derived cheaters resulting from evolution experiments (2) or mutagenesis $(28,29)$ can cause major population collapses due to cheating load (30-32), sometimes driving entire populations they inhabit to extinction (8). Analogous social collapse due to conspecific social parasitism has been documented in the African honeybee (33).

Cheaters that emerge in nature therefore have the theoretical potential to devastate the populations in which they arise. However, it has been hypothesized that high levels of genetic diversity present in even small-scale soil populations of $M$. xanthus $(23,34)$ may limit the spatial spread of such cheaters through positively frequency-dependent antagonisms (34). Such antagonisms occur pervasively among developmentally proficient natural isolates and are often lethal and expressed during both vegetative growth and starvation-induced development.

Several cheater genotypes have been studied in $M$. xanthus $(2,35)$, including some with characterized developmental defects. For example, mutations in the genes asgB (36) and csgA (29) prevent mutants from producing signal molecules (A-signal and C-signal, respectively) necessary for the early stages of fruiting body formation, and both ultimately reduce spore production in homogeneous groups by several orders of magnitude (2). Here we focus on the most-studied M. xanthus cheater, DK5208 (also known as LS523, see Methods), which has a transposon insertion in $\operatorname{csg} A$ (29). While this gene is necessary for normal development, the precise mechanisms of $\mathrm{C}$-signaling are debated. Earlier research suggested that the Csignal is a 17-KD fragment of CsgA which acts as an outer membrane signal and impacts developmental timing (37-39), while more recent studies suggest that the signal may derive from lipids generated by CsgA phospholipase activity in starving cells (40).

To date, $M$. xanthus cheaters have been studied primarily in the social context of pairwise interactions with their cooperative parent or recent ancestor. Here we used the $M$. xanthus $\operatorname{csgA}$ mutant DK5208 to investigate the role of allopatric divergence in cheating. We investigate the ability of the cheater to cheat on apparently allopatrically diverged natural strains. We further ask whether allopatric divergence in environments in which a cooperative trait is not expressed can latently generate barriers to social exploitation in a different environment in which the trait is expressed by testing the cheater's ability to exploit closely-related descendants of its cooperative parent. In this scenario, evolution happening outside of the environments that induce the cooperative trait and in the absence of the cheaters that exploit it creates social contexts in which the cheater is unable to cheat upon secondary contact, thereby constricting its cheating range and limiting its spread $(41,42)$.

Social phenotypes such as cheating may be limited not only by environmental and social context (43) but also by genomic context. We therefore asked whether the effect of disrupting a cooperation gene on development-related phenotypes can depend on epistatic interactions between the resulting allele and the rest of the genome. To do so, we disrupted $\operatorname{csg} A$ in the same manner in several cooperative natural isolates and tested for genetic background effects (44, 45), i.e. variation in the developmental phenotypes produced in pure culture and in mixtures with the parental isolates. Our collective results suggest that both the genomic context in which mutations arise and the social context in which they are expressed play more complex roles in determining the fate of noncooperation alleles in cooperative biological systems than has been previously understood.

\section{Results}

Cheating range excludes genetically distant natural isolates. DK5208 is known to cheat on the developmentally proficient lab strain GJV1 during starvation by converting a greater proportion of its vegetative cells into spores than does GJV1 in mixed groups despite producing far fewer spores than GJV1 in monoculture (2). The two strains are closely related; GJV1 is a sub-cultured recent descendent of DK1622 (46), the strain from which DK5208 was created (see Methods). However, it has been hypothesized that inter-strain antagonisms may restrict the range of cooperation-proficient genotypes on which a given cheater can successfully cheat (34). To test this, we mixed DK5208 in the minority (1:99) with a diverse array of natural strains isolated from various locations around the world $(47,48)$ just prior to starvation. We compared spore production by DK5208 in mixes with GJV1 versus with the 23 natural isolates, which are generally expected to differ from GJV1 by at least tens of thousands of mutations (Fig. 1 in 48, Fig. S2 in 49). As expected, DK5208 cheated on GJV1 ( $p=0.005$, one-sided $t$-test for $W_{i j}>0$ ). However, not only did DK5208 fail to cheat on any of the natural isolates (mean $W_{i j}$ values $<0, p$-values $<0.005,23$ two-sided $t$ - 


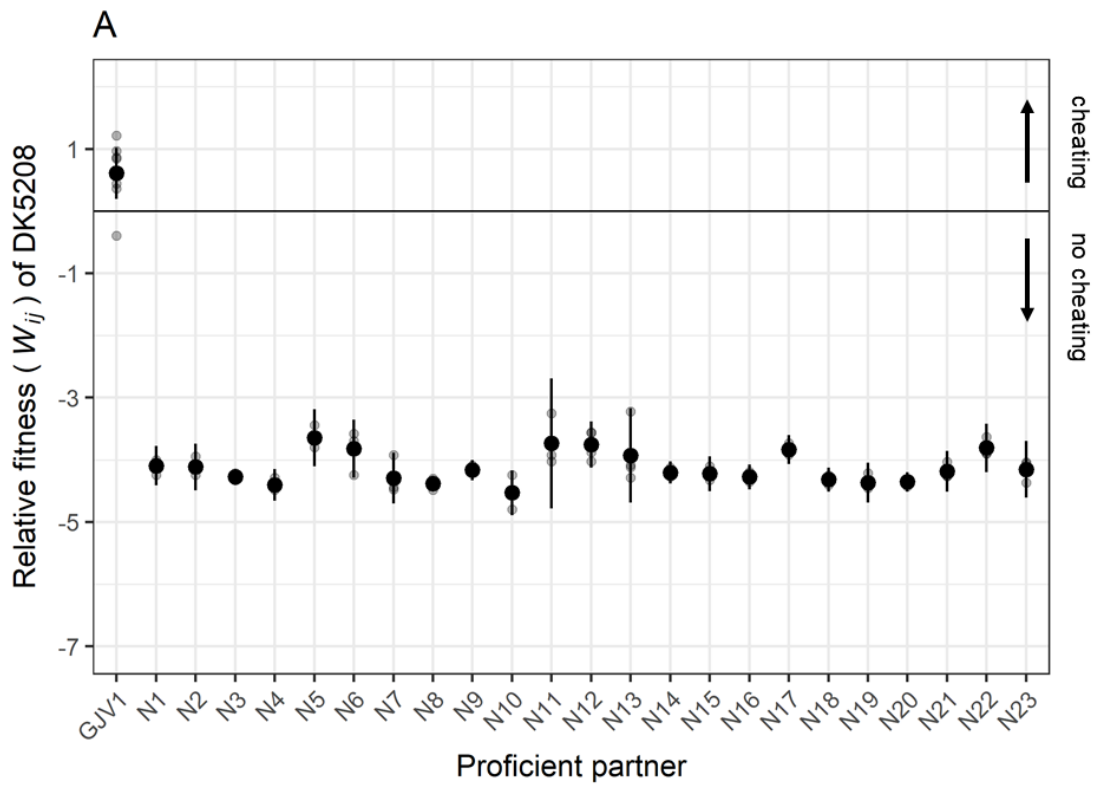

Figure 1. DK5208 cheats on a closely related cooperator but is outcompeted by distantly related natural isolates. Sporulation fitness $\left(W_{i j}\right)$ of DK5208 relative to GJV1 and 23 natural isolates in initially 1:99 (DK5208:competitor) mixed cultures. Grey circles represent individual replicate estimates, black circles show cross-replicate averages, and error bars show $95 \%$ confidence intervals; 3 or 4 biological replicates for natural isolate mixes, 8 for GJV1.

tests against 0 with Bonferroni-Holm correction; Fig. 1), it produced zero or extremely few spores in all pairings (Fig. S1A).

To test whether cheater-independent interactions, rather than any cheater-specific mechanism, were responsible for preventing DK5208 from sporulating in the preceding experiment (as expected), we mixed a developmentally proficient, rifampicin-resistant variant of GJV1 (GJV2) with the natural isolates (Fig. S1B). Although we observed a greater negative marker effect on GJV2 sporulation than observed in prior studies (25, $50)$, GJV2 nonetheless produced substantial numbers of spores in mixture with GJV1. However, like DK5208, it produced none (or almost none) when mixed with the natural isolates. The failure of GJV2 to sporulate in these mixtures indicates that DK5208 is indeed prevented from cheating on these strains by mechanisms that do not specifically target cheaters.

\section{Small degrees of allopatric divergence can} eliminate cheating upon secondary contact. Since DK5208 was suppressed in mixtures with genetically distant natural isolates, we tested whether smaller degrees of divergence might also reduce or eliminate DK5208's ability to cheat. We performed additional developmental competitions using developmentallyproficient clones from nine experimental populations that descended from GJV1 in an evolution experiment recently named MyxoEE-3 $(51,52)$. The MyxoEE-3 populations examined here evolved in nutrient-rich environments in which starvation-induced cooperative development was not expressed and so were not under selection to improve fitness during development. These populations also evolved in the absence of DK5208 (or any other known cheater) and therefore had no opportunity to interact in an evolutionarily relevant way with any genotypes capable of developmental cheating (51). Clones isolated from these MyxoEE-3 populations had each accumulated no more than 20 mutations (51).

As expected from previous experiments, DK5208 cheated on GJV1 (Figs. 2 and S2; $p=0.016$, one-sided $t$-test for $\left.W_{i j}>0\right)$. However, like the natural isolates, evolved MyxoEE-3 clones exhibited a clear trend of decreased susceptibility to cheating. DK5208 relative fitness estimates were lower against eight of the nine evolved clones than against GJV1 (all except E6, Fig. 2 ), an outcome unlikely to have occurred by chance (one-tailed sign test, $p=0.02$ ). This trend could be viewed as surprising; one might have expected these MyxoEE-3 lineages to have latently evolved reduced fitness compared to the cheater under starvation conditions because they underwent adaptation for many generations only in nutrient-rich experimental habitats - adaptation that might have traded off against fitness during starvation.

DK5208 had much lower fitness against two evolved clones in particular - E2 and E4 - than against the ancestor GJV1 ( $p$-values < 0.001, Dunnett test for difference from GJV1). DK5208 not only failed to outcompete E2 and E4 (Figs. 2 and S2; $p$-values $>0.1$, 9 two-sided $t$-tests against 0 with Bonferroni-Holm correction) but in fact appears to be outcompeted by them, as it was by the natural isolates (mean and 95\% confidence interval of $W_{i j}=-0.7[-1.2,-0.3]$ and $-2.1[-$ 3.6, -0.6], respectively). Thus, the ten mutational steps each which separate E2 and E4 from GJV1 (Table S2) 


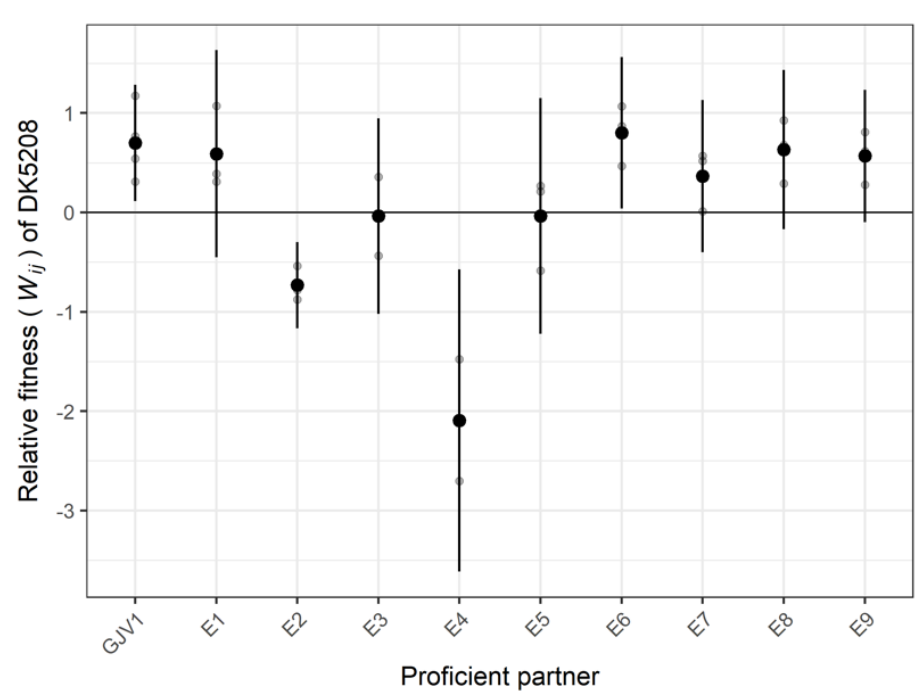

Figure 2. DK5208 cheats on GJV1 but not on some of GJV1's closely related descendants. Sporulation fitness $\left(W_{i j)}\right.$ of DK5208 relative to GJV1 and descendants of GJV1 from MyxoEE-3 $\left(W_{i j}\right)$ in initially 1:99 (DK5208:competitor) mixed cultures. Symbols as in Fig. 1; 3-4 biological replicates.

are enough to alter the fitness ranks emerging from this social interaction. This illustrates that even small degrees of allopatric, cheater-blind evolution in environments in which a focal cooperative trait is not expressed can latently generate resistance to cheating in environments in which cooperation does occur.

\section{Sporulation phenotypes are subject to epistatic} effects on $\operatorname{csg} A$ disruption. We then sought to test whether genomic background can also determine whether a potential cheating phenotype is expressed. To compare fitness effects of a $\operatorname{csg} A$ mutation across genomic backgrounds, we introduced an identical disruption of this gene into four of the natural isolates as well as GJV1. Specifically, we constructed plasmids able to integrate into each strain at $\operatorname{cs} g A$ by amplifying a 370-bp internal fragment of each strain's allele using primer sequences within $\operatorname{csg} A$ that are fully conserved across all strains (Fig. S3A) and ligating each fragment into the pCR-Blunt plasmid vector (Table S3). Transformation with the resulting plasmids successfully disrupted $\operatorname{csg} A$ at base-pair position 413 (out of $690 \mathrm{bp}$, see Methods) in all five strains and created partial-copy merodiploids at that locus.

The five resulting $\operatorname{csg} A$ mutants vary greatly in their developmental phenotypes despite carrying the same mutation, indicating that phenotypic expression of this mutation is subject to strong epistatic effects. All four natural-isolate mutants produced far fewer spores than their parental strains in monoculture (max. $~ 1 \%$ of parent; $p<0.032$ for the four natural isolates, five onesided $t$-tests against 0 with Bonferroni-Holm correction; Fig. 3). Three natural-isolate mutants (N9 csgA, N16 $\operatorname{csg} A$, and $\mathrm{N} 23 \operatorname{csg} A$ ) produced intermediate numbers of spores, while N2 $\operatorname{csg} A$ showed complete elimination of spore production (Fig. S4). However, the mutation had no significant effect on spore production in the GJV1 background ( $p=0.26$, same $t$-tests as above).

These outcomes indicate that different genomic backgrounds require different lengths of uninterrupted $\operatorname{csg} A$ to achieve high levels of sporulation; 413 base pairs of the GJV1 allele was sufficient in the GJV1 background, but the same length of the N2 allele was insufficient in the N2 background. Thus, we find that an identical mutation can have vastly different effects on a social phenotype depending on the genomic context in which it occurs. Genomic divergence among cooperators (presumably in allopatry; Table S1) altered the genetic requirements for expression of a cooperation-based trait - in this case, the length of intact $\operatorname{csg} A$ gene required to allow high levels of spore production.

The lack of effect of our engineered csgA disruption in GJV1 contrasts with the strong negative effect of the $\operatorname{csgA}$ mutation carried by DK5208 $(p=0.0003$, paired ttest for difference between DK5208 and GJV1 csgA, df $=3$, mean of log-differences with $95 \%$ confidence interval $=6.5[5.4,7.5]$; Fig. 3). We hypothesize that this is due to the difference in lengths of partial CsgA fragments produced by the two mutants. Our plasmid disrupts $\operatorname{csgA}$ closer to its 3 ' end (position 413 of 690 bp) than does the transposon insertion in DK5208 (position 217 (53)) and permits sufficient gene activity to sporulate at the parental level in the DK1622/GJV1 background.

Because the new $\operatorname{csg} A$ disruption does not reduce GJV1 spore production, it does not, by definition, have the potential to generate an obligately defective cheating phenotype in that background. However,

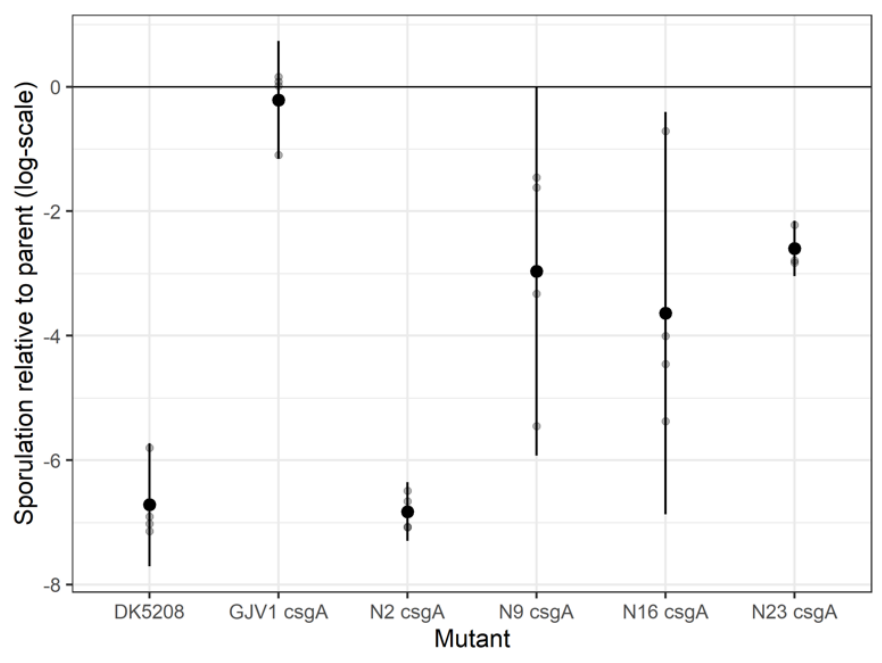

Figure 3. Genetic background effects on the phenotype of a csgA mutation. We show the pure-culture spore production of each new $\operatorname{csg} A$ mutant relative to that of its parent. The DK5208 csgA transposon mutation severely reduces spore production relative to GJV1, whereas the new $\operatorname{csg} A$ plasmid-disruption mutation in the GJV1 background does not. Genetic background effects: The same $\operatorname{csg} A$ plasmid disruption impacts relative spore production very differently depending on the parental genotype, thus revealing strong epistatic interactions and developmental system drift. Symbols as in Fig. 1; 4 biological replicates. 
since the other mutants exhibited significant defects, we could test whether they cheated on their parent strains. The defects of all four natural-isolate $\operatorname{csg} A$ mutants were fully complemented by their own parental strains in mixed groups (Figs. 4, S4 and S5). However, we detected no significant differences in relative fitness for any of the mutant-parent strain pairs ( $p$-values > $0.05,26$ two-sided t-tests against 0 with BonferroniHolm correction; Fig. 4). In other words, these mutants socially exploited their parents for gains in absolute fitness that fully compensated for their defects, but these gains were not high enough to be considered cheating, at least at the initial frequencies and under the environmental conditions examined here.

We also tested whether these mutants would fare poorly when mixed in the minority with cooperators that are highly diverged from their own parent. This outcome was expected because developmental fitness in competitions between developmentally-proficient natural isolates tends to be frequency dependent (34), with minority genotypes almost always losing to majority genotypes irrespective of fitness outcomes in 1:1 mixes. Just as the $\operatorname{csg} A$ cheater DK5208 was unable to exploit any natural isolate for gains in absolute fitness (Fig. S1A), the plasmid-disruption $\operatorname{csg} A$ mutants were also generally not complemented in mixes with cooperation-proficient strains other than their own parent. Indeed, in most cases, the mutants produced either no spores or extremely few in mixture with nonparental strains (Fig. S4). One surprising exception was the mixture of $\mathrm{N} 16 \operatorname{csg} A$ with $\mathrm{N} 23$, in which the mutant was neither significantly harmed nor complemented ( $p=0.51,26$ two-sided $t$-tests against 0 with Bonferroni-Holm correction), indicating that, independent of the $\operatorname{csg} A$ mutation, the N16 background has high enough fitness relative to N23 to compensate for being in the minority in mixture.

\section{Discussion}

We examined the role of allopatric divergence in the expression and potentiation of cheating phenotypes in $M$. xanthus, whether it manifests in the effect of the social partner on the cheater or in the influence of the mutant's genomic background on the effect of the mutation.

Regarding social context, expression of a well-known cheating phenotype appears to depend on how closely related a cooperation-defective mutant (DK5208) is to the developmentally proficient cooperators with which it interacts. Although DK5208 cheats on a strain (GJV1) that is nearly identical to its parent (DK1622), it does not merely fail to cheat when mixed with genetically distant natural isolates (Fig. 1) but effectively fails to sporulate at all (Fig. S1A). The extremely low fitness of this cheater against the natural isolates appears unrelated to the developmental defect that allows it to cheat on GJV1. This is because sporulation by two different sporulation-proficient mutants of GJV1 - GJV2 and GJV1 $\operatorname{csg} A$ - is also effectively eliminated by the same natural isolates in mixed competitions (Figs. S1B and S4). These antagonisms are likely due to toxin production that is not specific to development, as such antagonism between natural isolates often occurs during both growth and development (34). Our experiments show that social (or intergenomic) epistasis $(54,55)$ in pairwise interactions between cooperators and a cheater can be either positive or negative, in this case allowing cheating in one pair but suppressing it in all others, due to divergence among cooperators that was apparently not specific to the cooperative interaction of $\mathrm{C}$-signaling.

This result with natural isolates led us to ask how readily social barriers to cheating might evolve in cooperative lineages in environments in which they do not even express a cheatable cooperative trait and do not meet cheaters at all. That cheater resistance might emerge in such a fashion was plausible in light of other novel social interactions known to evolve indirectly, including obligate cheating (2), facultative social exploitation (25), and kin discrimination in the form of colony-merger incompatibilities (24).

When mixed with clones from the MyxoEE-3 evolution experiment (closely related lab-evolved cooperators that had no history of experimental selection on development because they evolved in nutrient-rich environments and in the absence of the cheater), our focal cheater DK5208 exhibited a trend of reduced fitness compared to mixture with the experimental ancestor, and it failed to cheat on multiple evolved cooperators (Fig. 2). That this trend would evolve was not obvious, as we might have expected the evolved strains to have actually decreased in fitness relative to the cheater during MyxoEE-3 due to the combination of a lack of selection on traits related to starvation and the presence of selection for adaptation to resource abundance.

The evolutionary changes in some lab-evolved cooperators during MyxoEE-3 that prevent DK5208 from cheating on them appear unrelated to the defect in C-signaling which allows DK5208 to cheat on the ancestral cooperator. This inference derives from the known absence of experimental selection on development in MyxoEE-3 and is strengthened by the absence of mutations in $\operatorname{cs} g A$ in the genomes of several of the MyxoEE-3 clones examined here (E1-E5; 24). These include the two clones that most strongly inhibit cheating by DK5208 (E2 and E4). Collectively, our results with both lab-evolved and natural-isolate cooperators demonstrate that a cheating phenotype can be nullified by very small degrees of diversification ( $\leq 10$ mutations; Table S1) happening outside the context of cooperation and in the absence of cheaters and suggest that the strength of a strain's cheater resistance may increase with evolutionary distance from the cheater, at least in a broad sense.

These outcomes further suggest that cheaters arising in spatially structured natural populations $(34,47,49$, $56,57)$ are likely to have cheating ranges within which they can advantageously exploit cooperators 


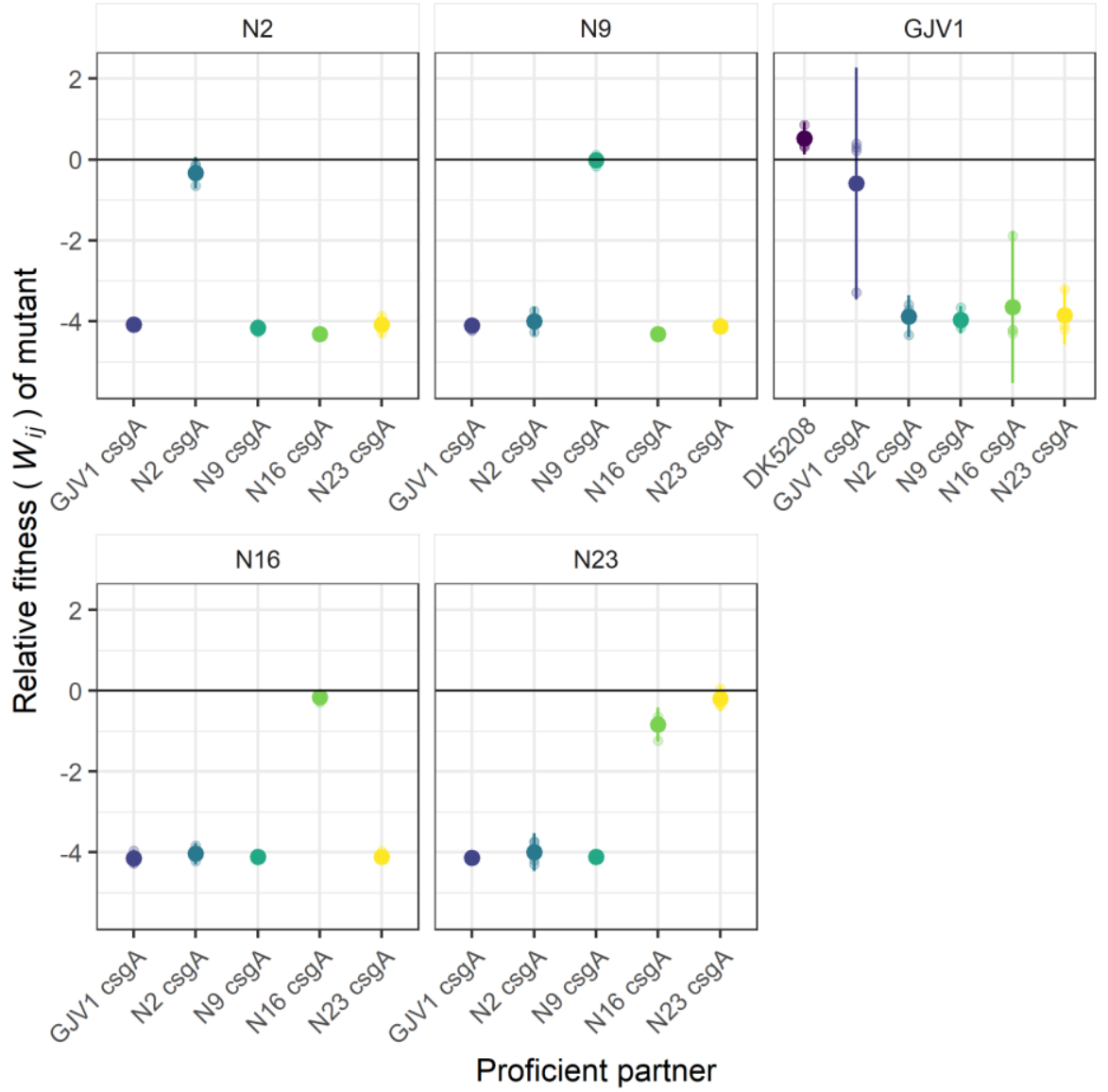

Figure 4. New $\operatorname{csg} A$ mutants have relative fitness similar to their parent in mixed groups but are generally outcompeted by non-parental cooperators. Sporulation fitness $\left(W_{i j}\right)$ of $\operatorname{csg} A$ mutants relative to developmentally proficient partners in initially 1:99 (mutant:partner) mixed groups. Symbols as in Fig. 1; 4 biological replicates.

(analogous to parasite host ranges (58-60)) that are narrow both genetically and, because genetic and spatial distance correlate in wild populations (61), geographically. Cheater-blind allopatric divergence, emerging due to drift or selection, may generate a patchy phylogeographic mosaic of cheater-cooperator compatibility types across which most cooperator genotypes are resistant to most cheater genotypes from other patches. Such indirect barriers to cheating might be reinforced by unique local patterns of cooperator-cheater coevolution (4, 18, 23, 62-64). Avenues for future research include i) investigating the geographic and genetic scale of cooperator-cheater compatibility patches in natural populations and ii) incorporating the potential for cheater-blind barriers to cheating into models of spatially structured social evolution (65), in particular when considering relative contributions of migration vs other forces in shaping equilibrium levels and biogeography of cooperation and cheating.

We propose that reduction in the efficacy of cheating between cheaters and conspecific cooperators due to allopatric divergence may be common across a broad range of social systems (e.g. other social microbes and social animals). However, the mechanisms by and evolutionary rapidity with which allopatrically-evolved forms of kin discrimination $(24,25)$ hinder cheating are likely to be highly variable across systems, and they may be influenced by the complexity of the cooperative trait being cheated on and the degree of physical proximity between interactants during the behavior. $M$. xanthus cells produce many and diverse extracellular compounds during both growth and development (26), alteration of which may provide many mechanistic routes to reduce social compatibility between cells of allopatrically diverged cheaters and cooperators that come into close physical contact during aggregative development. It may be that simpler cooperative behaviors, such as production of siderophores that can diffuse to conspecifics at a distance (17), are less likely to be protected from cheating by generic evolutionary divergence among strains. However, it may still be possible for various siderophores and their receptors (18), and likewise quorum-sensing or other signals together with their receptors, to diverge in allopatry and thereby generate a biogeographic patchwork of cheating ranges and efficacies.

Regarding genomic context, fruiting body development and sporulation in $M$. xanthus are complex processes involving many genes and regulatory pathways $(26,66-68)$. Evolutionary change that affects genes contributing to a complex 
developmental process can alter their epistatic relationships and thereby generate genetic background effects (GBEs), in which an allele produces different phenotypes in different genetic backgrounds $(44,45)$. GBEs have been found in many systems, including insects $(69,70)$, maize (71), mice (72), and microbes $(73,74)$. We tested for GBEs on the cheating phenotype considered here by disrupting the developmental signaling gene $\operatorname{csg} A$ in a set of distinct, well-diverged strains. This disruption produced highly variable reductions of pure-culture spore production across the four natural isolates - in one case (N2) effectively eliminating sporulation - but left spore output at a wild-type level in the GJV1 background. These strains have thus diverged in how their genetic backgrounds epistatically interact with a disruption that causes the loss of the terminal portion of $\operatorname{csg} A$. The 5' region of $\operatorname{csg} A$ left intact upstream of the disruption provides sufficient function to allow normal sporulation in the GJV1 background but not in the natural isolates. Genomic divergence between N2 and GJV1 altered the length of intact $\operatorname{csg} A$ necessary for cooperative spore production.

This outcome has both evo-devo and social-evolution implications. In aggregative multicellular systems, evodevo $(75,76)$ and social evolution are intrinsically intertwined because development is a cooperative process among reproductively autonomous organisms. From an evo-devo perspective, the csgA-disruption results demonstrate developmental system drift (DSD; 77) at the intraspecific scale in a microbial system. In DSD, the genetic basis of a developmental phenotype diverges across lineages, either stochastically (77) or due to selection $(44,78)$, while the fundamental phenotype itself is conserved. This phenomenon has been documented in a wide range of systems (79-81). In the myxobacteria, the gene sets necessary for fruiting body development have diverged extensively across species $(82,83)$. At much shorter evolutionary timescales, analyses of an experimental lineage (84) and natural variation at a regulatory region (48) have shown that the genetic pathways underlying $M$. xanthus development are evolutionarily malleable (85). Our $\operatorname{csg} A$-disruption results show that the general epistatic environment of these pathways can diverge sufficiently within the same species to render a gene or part of a gene conditionally essential to a major developmental phenotype (86).

From a social evolution perspective, diversification generated epistatic effects on the phenotype caused by disruption of a key cooperation gene. Identical disruption of $\operatorname{csg} A$ in divergent genomic backgrounds shows that whether a given mutation generates a social defect required for obligate cheating can depend on genomic context. This suggests that different strains of M. xanthus may differ in their sets of mutations that confer a cheating phenotype, which could limit the ability of any given cheating mutation arising in one genomic background to spread across genomic backgrounds by horizontal gene transfer. Thus, in aggregatively multicellular microbes, just as social selection can drive evolution of developmental features $(12,84,87)$, so too may divergence of developmental genetic architecture reciprocally shape social evolution, including which mutations generate defects in cooperative traits.

\section{Materials and Methods}

Semantics. In this study, we use 'obligate cheating' (or simply 'cheating') to refer to a social interaction in which one interactant, the cheater, is obligately defective to some extent at expressing a focal cooperative trait relative to a cooperative genotype, and yet gains a relative fitness advantage over the cooperator by social exploitation when they interact under relevant conditions (e.g. 8, 10). Here we do not consider strains that are intrinsically proficient at a high level of cooperation yet outcompete other cooperationproficient strains in mixed groups during a cooperative process (which are sometimes called 'facultative cheaters' (14) or 'facultative exploiters' (25)), as their competitive success does not undermine the persistence of cooperation per se. We use 'social exploitation' to refer to any social interaction from which a focal partner derives an absolute-fitness benefit, even if the other interactant(s) are not harmed (88). Finally, we note that 'allopatric divergence' in the article title applies somewhat differently to our results using MyxoEE-3 clones and those using natural isolates. 'Allopatric' applies with certainty to MyxoEE-3 clones relative to DK5208, as it is known that evolving populations never interacted with DK5208 (or any other cheater during development) during MyxoEE-3. 'Allopatric' applies with only high probability to the natural isolates. Their detailed evolutionary histories since divergence are unknown, but because they were isolated from locations very distant from where the natural-isolate progenitor of DK1622/GJV1 was isolated (Ames, lowa; 89) and, for the parents of the mutants highlighted in Fig. 4, from one another, we consider the probability that their ancestral lineages directly interacted for evolutionarily significant periods to be very small.

Strains and growth conditions. As a focal cheater strain, we used the developmental mutant DK5208 (Table S1). DK5208 is a yellow clonal isolate of the strain LS523 $(4,90)$. LS523 was derived from the wildtype strain DK1622 and contains a Tn5 transposon insertion in the csgA gene at position 217 out of $690 \mathrm{bp}$ which confers resistance to oxytetracycline $(29,53)$. As developmentally proficient cooperative strains, we used (i) the wild-type strain GJV1 (strain 'S' in 50), which is derived from DK1622, the strain from which DK5208 was created (29), via a small degree of lab subculturing and which differs from DK1622 by only five mutations (46); (ii) nine experimentally-evolved clones descended from GJV1 during MyxoEE-3 which retained high levels of spore production, (iii) thirteen 
natural isolates from around the world, and (iv) ten natural isolates from Bloomington, Indiana, USA (see Table S1). The experimentally evolved clones were isolated from populations descending from either GJV1 or a rifampicin-resistant variant of GJV1 (GJV2) and which had undergone 40 two-week cycles of evolution as motile colonies expanding on $0.5 \%$ or $1.5 \%$ nutrient agar, as described previously $(24,25,51)$. In experiments testing whether the effects we observed were specific to the cheater strain, we used GJV2, a spontaneous rifampicin-resistant mutant of GJV1 (aka strain ' $R$ ' in 50 , see also 12,91 ).

We inoculated frozen $M$. xanthus stocks onto CTT (92) $1.5 \%$ agar plates and incubated at $32{ }^{\circ} \mathrm{C}$ and $90 \%$ $\mathrm{rH}$ for 4-5 days, after which we inoculated colony samples into CTT liquid and grow them overnight at 32 ${ }^{\circ} \mathrm{C}$ with shaking at $300 \mathrm{rpm}$. Where appropriate, we grew strains in media containing $40 \mu \mathrm{g} / \mathrm{ml}$ kanamycin or $5 \mu \mathrm{g} / \mathrm{ml}$ oxytetracycline.

Plasmids and mutant construction. We constructed novel csgA (NCBI DK1622 locus tag MXAN_RS06255, old locus tag MXAN_1294) mutants of $\bar{M}$. xanthus strains GJV1, Chihaya 20, Serengeti 01, GH3.5.6c2, and MC3.5.9c15 by disrupting the gene with a plasmid insertion. To construct the plasmids, we selected primer sequences for PCR which were in conserved regions of $\operatorname{csg} A$ by aligning the $\operatorname{csg} A$ sequence of the published $M$. xanthus DK1622 genome (NCBI:txid246197) with the genomes of the natural isolates (SRA accession numbers: SRR8298023, SRR8298022 (48, 49); Fig. S3A). Phylogenetic relationships among the $\operatorname{csg} A$ sequences are shown in Fig. S3B. So that each strain could be transformed with a plasmid containing a fragment of its own $\operatorname{csg} A$ allele, we PCR-amplified a 370-bp internal fragment of $\operatorname{csg} A$ from each of the natural isolates and GJV1 using forward primer TAATTCGTCCAGCAGCTCCTGCTGC-3' (csgA positions 44-67, genome positions 1520242-1520265 (bold text)), and reverse primer 5'TTACCCATCCGCGAGGTGACGTG-3' (csgA positions 394-413, genome positions 15205921520611 (bold text)). We purified the fragments using the QIAquick® PCR Purification Kit (QIAGEN, Hilden, Germany) and verified their length on a $1 \%$ agarose gel, then ligated each insert into the pCR-Blunt vector (Invitrogen, San Diego, CA), which carries a kanamycin resistance marker. We verified the pCR-csgA413 plasmids (Table S3) bearing the individual $\operatorname{csg} A$ fragments by sequencing (Microsynth AG, Balgach, Switzerland). We electroporated each M. xanthus strain with the respective plasmid to create merodiploids with truncated copies of $\operatorname{csg} A$. The CsgA protein encoded by the first partial $\operatorname{csg} A$ sequence is truncated at its amino acid position 138 by the plasmid integration. The second copy is deprived of the native csgA promoter and its 5' terminal sequence contains a stop codon engineered at the beginning of the amplified $\operatorname{csg} A$ fragment. Thus, successful transformants are designed to produce a truncated CsgA protein only 137 aa long, or $\sim 60 \%$ of the full-length 229 -aa GJV1 CsgA protein. We verified transformants by diagnostic PCR and antibiotic-resistance phenotype.

Developmental assays. We centrifuged exponentialphase liquid cultures at $5000 \mathrm{rpm}$ for 15 minutes and resuspended in nutrient-free TPM (66) $\mathrm{pH} 8.0$ to a density of $5 \times 10^{9} \mathrm{cells} / \mathrm{ml}$. We inoculated $50 \mu \mathrm{l}$ of each strain or mixture onto TPM pH $8.01 .5 \%$ agar plates to initiate development. We made all mixes at a 1:99 ratio by mixing $1 \mu \mathrm{l}$ of the $\operatorname{csg} A$ mutant and $99 \mu \mathrm{l}$ of the cooperator. We incubated the TPM plates at $32{ }^{\circ} \mathrm{C}$ and $90 \%$ relative humidity for 72 hours. We then harvested each entire starved population from the plates with sterile scalpels and incubated each sample in $1 \mathrm{ml}$ of $\mathrm{ddH}_{2} \mathrm{O}$ for 2 hours at $50{ }^{\circ} \mathrm{C}$ to kill any non-spore cells. We dispersed the spores by sonicating twice for 20 seconds with a 10 second pause. We dilution-plated the samples into CTT $0.5 \%$ agar to count the number of CFUs. For mixed competitions, we plated the samples into agar with and without antibiotic to generate counts for the $\operatorname{csg} A$ mutants and for the mixture as a whole, respectively, and therefore by subtraction counts for the cooperators. We plated pure-culture samples of the $\operatorname{csg} A$ mutants with antibiotic.

Data analysis. We performed all data analysis and statistical testing using $R$ version 4.0.0 and RStudio version 1.2.5042, and we visualized the data using the ggplot2 package (93). All original data files and analysis protocols, including statistical scripts, R Markdown files, and full results of statistical tests, may be accessed via Dryad doi: $10.5061 /$ dryad.fbg79cnsb. For the $\operatorname{csg} A$ mutants in the developmental assays, we calculated the one-way mixing effect

$$
C_{i}(j)=\log \left(\frac{\text { sporulation ef ficiency in mixture }}{\text { sporulation ef ficiency in pure culture }}\right)
$$

where "sporulation efficiency" refers to the fraction of cells inoculated that became spores, and the relative fitness in mixture

$$
W_{i j}=\log \left(\frac{\text { sporulation efficiency of mutant }}{\text { sporulation ef ficiency of cooperator }}\right)
$$

as in Vos and Velicer (23). Here, the $i$ refers to the mutant and the $j$ refers to the cooperator. We analyzed our experimental results using general linear models followed by post-hoc Tukey HSD tests, Dunnett tests, or $t$-tests, as reported in the results. For calculations of $C_{i}(j)$ and $W_{i j}$, we assumed that strains for which we counted zero spores produced the maximum number of spores which would have been below the detection limit of our dilution plates.

We constructed the phylogeny in Figure S3B using Clustal Omega version 1.2.1 (94), PhyML version 3.1 (95), and Newick Display (96) via the Galaxy platform (galaxy.pasteur.fr; (97)). 


\section{Acknowledgements}

We thank Sébastien Wielgoss for assistance with the natural isolate sequence data, Gilles Pütz, Ralph Silva, and Zachary Blount for productive discussions, and two anonymous reviewers for helpful comments. This study was supported in part by Swiss National Science Foundation (SNSF) grants 31003A/B_16005 to GJV and an ETH Fellowship to MV.

\section{References}

1. J. A. Fletcher, M. Doebeli, A simple and general explanation for the evolution of altruism. Proc. $R$. Soc. B Biol. Sci. 276, 13-19 (2009).

2. G. J. Velicer, L. Kroos, R. E. Lenski, Developmental cheating in the social bacterium Myxococcus xanthus. Nature 404, 598-601 (2000).

3. A. Gardner, S. A. West, Greenbeards. Evolution 64, 25-38 (2010).

4. P. Manhes, G. J. Velicer, Experimental evolution of selfish policing in social bacteria. Proc. Natl. Acad. Sci. 108, 8357-62 (2011).

5. J. B. Xavier, W. Kim, K. R. Foster, A molecular mechanism that stabilizes cooperative secretions in Pseudomonas aeruginosa. Mol. Microbiol. 79, 166-179 (2011).

6. A. J. Waite, W. Y. Shou, Adaptation to a new environment allows cooperators to purge cheaters stochastically. Proc. Natl. Acad. Sci. U. S. A. 109, 19079-19086 (2012).

7. R. C. Allen, L. McNally, R. Popat, S. P. Brown, Quorum sensing protects bacterial co-operation from exploitation by cheats. ISME J. 10, 17061716 (2016).

8. F. Fiegna, G. J. Velicer, Competitive fates of bacterial social parasites: persistence and selfinduced extinction of Myxococcus xanthus cheaters. Proc. R. Soc. B 270, 1527-34 (2003).

9. K. R. Foster, G. Shaulsky, J. E. Strassmann, D. C. Queller, C. R. L. Thompson, Pleiotropy as a mechanism to stabilize cooperation. Nature 431, 693-6 (2004).

10. M. Travisano, G. J. Velicer, Strategies of microbial cheater control. Trends Microbiol. 12, 72-78 (2004).

11. M. A. Brockhurst, M. E. Hochberg, T. Bell, A. Buckling, Character Displacement Promotes Cooperation in Bacterial Biofilms. Curr. Biol. 16, 2030-2034 (2006).

12. F. Fiegna, Y. N. Yu, S. V Kadam, G. J. Velicer, Evolution of an obligate social cheater to a superior cooperator. Nature 441, 310-314 (2006).

13. J. S. Chuang, O. Rivoire, S. Leibler, Simpson's Paradox in a Synthetic Microbial System. Science 323, 272-275 (2009).

14. A. Khare, et al., Cheater-resistance is not futile. Nature 461, 980-982 (2009).

\section{Author contributions}

KAS and GJV designed the experiments; KAS, YTNY, and GJV designed the mutants; KAS carried out the experiments; KAS and MV analyzed the data; KAS, YTNY, MV, and GJV wrote the manuscript.

15. A. A. Smith, B. Hölldober, J. Liebig, Cuticular Hydrocarbons Reliably Identify Cheaters and Allow Enforcement of Altruism in a Social Insect. Curr. Biol. 19, 78-81 (2009).

16. A. D. Morgan, B. J. Z. Z. Quigley, S. P. Brown, A. Buckling, Selection on non-social traits limits the invasion of social cheats. Ecol. Lett. 15, 841-846 (2012).

17. R. Kümmerli, et al., Co-evolutionary dynamics between public good producers and cheats in the bacterium Pseudomonas aeruginosa. J. Evol. Biol. 28, 2264-2274 (2015).

18. E. Butaite, M. Baumgartner, S. Wyder, R. Kümmerli, Siderophore cheating and cheating resistance shape competition for iron in soil and freshwater Pseudomonas communities. Nat. Commun. 8 (2017).

19. T. Dobzhansky, Genetics and the origin of species, Second ed. (Columbia University Press, 1950).

20. E. Mayr, Animal Species and Evolution (Harvard University Press, 1963).

21. B. Fischer, S. Foitzik, Local co-adaptation leading to a geographical mosaic of coevolution in a social parasite system. J. Evol. Biol. 17, 1026-1034 (2004).

22. C. A. Johnson, J. M. Herbers, Impact of parasite sympatry on the geographic mosaic of coevolution. Ecology 87, 382-394 (2006).

23. M. Vos, G. J. Velicer, Social Conflict in Centimeter- and Global-Scale Populations of the Bacterium Myxococcus xanthus. Curr. Biol. 19, 1763-7 (2009).

24. O. Rendueles, et al., Rapid and widespread de novo evolution of kin discrimination. Proc. Natl. Acad. Sci. 112, 9076-81 (2015).

25. R. R. Nair, F. Fiegna, G. J. Velicer, Indirect evolution of social fitness inequalities and facultative social exploitation. Proc. R. Soc. B Biol. Sci. 285 (2018).

26. J. Muñoz-Dorado, F. J. Marcos-Torres, E. GarcíaBravo, A. Moraleda-Muñoz, J. Pérez, Myxobacteria: Moving, killing, feeding, and surviving together. Front. Microbiol. 7, 1-18 (2016).

27. L. Kroos, Highly Signal-Responsive Gene Regulatory Network Governing Myxococcus Development. Trends Genet. 33, 3-15 (2017). 
28. A. Kuspa, L. Kroos, D. Kaiser, Intercellular signaling is required for developmental gene expression in Myxococcus xanthus. Dev. Biol. 117, 267-76 (1986).

29. L. J. Shimkets, S. J. Asher, Use of recombination techniques to examine the structure of the csg locus of Myxococcus xanthus. Mol. Gen. Genet. 211, 63-71 (1988).

30. J. D. Van Dyken, T. A. Linksvayer, M. J. Wade, Kin selection-mutation balance: A model for the origin, maintenance, and consequences of social cheating. Am. Nat. 177, 288-300 (2011).

31. A. J. Waite, C. Cannistra, W. Shou, Defectors Can Create Conditions That Rescue Cooperation. PLoS Comput. Biol. 11, 1-23 (2015).

32. C. Moreno-Fenoll, M. Cavaliere, E. MartínezGarcía, J. F. Poyatos, Eco-evolutionary feedbacks can rescue cooperation in microbial populations. Sci. Rep. 7, 42561 (2017).

33. B. P. Oldroyd, The Cape honeybee: An example of a social cancer. Trends Ecol. Evol. 17, 249-251 (2002).

34. O. Rendueles, M. Amherd, G. J. Velicer, Positively Frequency-Dependent Interference Competition Maintains Diversity and Pervades a Natural Population of Cooperative Microbes. Curr. Biol. 25, 1673-1681 (2015).

35. Y. T. N. Yu, M. Kleiner, G. J. Velicer, Spontaneous reversions of an evolutionary trait loss reveal regulators of a small RNA that controls multicellular development in myxobacteria. $J$. Bacteriol. 198, 3142-3151 (2016).

36. A. Kuspa, L. Plamann, D. Kaiser, A-signalling and the cell density requirement for Myxococcus xanthus development. J. Bacteriol. 174, 7360 7369 (1992).

37. L. J. Shimkets, H. Rafiee, CsgA, an extracellular protein essential for Myxococcus xanthus development. J. Bacteriol. 172, 5299-306 (1990).

38. S. Lobedanz, L. Søgaard-Andersen, Identification of the C-signal, a contact-dependent morphogen coordinating multiple developmental responses in Myxococcus xanthus. Genes Dev. 17, 2151-2161 (2003).

39. A. Rolbetzki, M. Ammon, V. Jakovljevic, A. Konovalova, L. Søgaard-Andersen, Regulated Secretion of a Protease Activates Intercellular Signaling during Fruiting Body Formation in $M$. xanthus. Dev. Cell 15, 627-634 (2008).

40. T. O. Boynton, L. J. Shimkets, Myxococcus CsgA, Drosophila Sniffer, and human HSD10 are cardiolipin phospholipases. Genes Dev. 29, 1903 1914 (2015).

41. A. Eldar, Social conflict drives the evolutionary divergence of quorum sensing. Proc. Natl. Acad. Sci. U. S. A. 108, 13635-13640 (2011).

42. I. Ben-Zion, S. Pollak, A. Eldar, Clonality and nonlinearity drive facultative-cooperation allele diversity. ISME J. 13, 824-835 (2019).

43. M. Ghoul, S. A. West, S. P. Diggle, A. S. Griffin,
An experimental test of whether cheating is context dependent. J. Evol. Biol. 27, 551-6 (2014).

44. C. H. Chandler, S. Chari, I. Dworkin, Does your gene need a background check? How genetic background impacts the analysis of mutations, genes, and evolution. Trends Genet. 29, 358-366 (2013).

45. M. N. Mullis, T. Matsui, R. Schell, R. Foree, I. M. Ehrenreich, The complex underpinnings of genetic background effects. Nat. Commun. 9, 1-10 (2018).

46. G. J. Velicer, et al., Comprehensive mutation identification in an evolved bacterial cooperator and its cheating ancestor. Proc. Natl. Acad. Sci. 103, 8107-8112 (2006).

47. S. A. Kraemer, G. J. Velicer, Endemic social diversity within natural kin groups of a cooperative bacterium. Proc. Natl. Acad. Sci. 108, 10823-30 (2011).

48. R. Rajagopalan, S. Wielgoss, G. Lippert, G. J. Velicer, L. Kroos, devl is an Evolutionarily Young Negative Regulator of Myxococcus xanthus Development. J. Bacteriol. 197, JB.02542-14 (2015).

49. S. Wielgoss, R. Wolfensberger, L. Sun, F. Fiegna, G. J. Velicer, Social genes are selection hotspots in kin groups of a soil microbe. Science 363 , 1342-1345 (2019).

50. G. J. Velicer, L. Kroos, R. E. Lenski, Loss of social behaviors by Myxococcus xanthus during evolution in an unstructured habitat. Proc. Natl. Acad. Sci. 95, 12376-80 (1998).

51. O. Rendueles, G. J. Velicer, Evolution by flight and fight: Diverse mechanisms of adaptation by actively motile microbes. ISME J. 11, 555-568 (2017).

52. O. Rendueles, G. J. Velicer, Hidden paths to endless forms most wonderful: Complexity of bacterial motility shapes diversification of latent phenotypes. BMC Evol. Biol. 20, 145 (2020).

53. B. U. Lee, K. Lee, J. Mendez, L. J. Shimkets, A tactile sensory system of Myxococcus xanthus involves an extracellular NAD(P)+-containing protein. Genes Dev. 9, 2964-2973 (1995).

54. T. A. Linksvayer, Ant species differences determined by epistasis between brood and worker genomes. PLoS One 2 (2007).

55. S. Teseo, N. Châline, P. Jaisson, D. J. C. Kronauer, Epistasis between adults and larvae underlies caste fate and fitness in a clonal ant. Nat. Commun. 5 (2014).

56. M. Vos, G. J. Velicer, Genetic population structure of the soil bacterium Myxococcus xanthus at the centimeter scale. Appl. Environ. Microbiol. 72, 3615-25 (2006).

57. S. A. Kraemer, S. Wielgoss, F. Fiegna, G. J. Velicer, The biogeography of kin discrimination across microbial neighborhoods. Mol. Ecol., 4875-4888 (2016). 
58. K. Holmfeldt, M. Middelboe, O. Nybroe, L. Riemann, Large variabilities in host strain susceptibility and phage host range govern interactions between lytic marine phages and their Flavobacterium hosts. Appl. Environ. Microbiol. 73, 6730-6739 (2007).

59. A. Best, et al., The evolution of host-parasite range. Am. Nat. 176, 63-71 (2010).

60. P. D. Scanlan, A. R. Hall, P. Burlinson, G. Preston, A. Buckling, No effect of host-parasite co-evolution on host range expansion. J. Evol. Biol. 26, 205-209 (2013).

61. M. Vos, G. J. Velicer, Isolation by Distance in the Spore-Forming Soil Bacterium Myxococcus xanthus. Curr. Biol. 18, 386-391 (2008).

62. J. N. Thompson, The Geographic Mosaic of Coevolution (University of Chicago Press, 2005).

63. Q.-G. Zhang, A. Buckling, R. J. Ellis, H. C. J. Godfray, Coevolution between cooperators and cheats in a microbial system. Evolution 63, 22482256 (2009).

64. H. R. Votaw, E. A. Ostrowski, Stalk size and altruism investment within and among populations of the social amoeba. J. Evol. Biol. 30, 2017-2030 (2017).

65. M. J. Wade, Adaptation in Metapopulations: How Interaction Changes Evolution (University of Chicago Press, 2016).

66. L. Kroos, A. Kuspa, D. Kaiser, A global analysis of developmentally regulated genes in Myxococcus xanthus. Dev. Biol. 117, 252-266 (1986).

67. L. Kroos, D. Kaiser, Expression of many developmentally regulated genes in Myxococcus depends on a sequence of cell interactions. Genes Dev. 1, 840-854 (1987).

68. D. Kaiser, M. Robinson, L. Kroos, Myxobacteria, polarity, and multicellular morphogenesis. Cold Spring Harb. Perspect. Biol. 2, 1-26 (2010).

69. J. A. McKenzie, M. J. Whitten, M. A. Adena, The effect of genetic background on the fitness of diazinon resistance genotypes of the australian sheep blowfly, Lucilia cuprina. Heredity (Edinb). 49, 1-9 (1982).

70. C. H. Chandler, S. Chari, D. Tack, I. Dworkin, Causes and Consequences of Genetic Background Effects Illuminated by Integrative Genomic Analysis. Genetics 196, 1321-1336 (2014).

71. S. Rio, et al., Disentangling group specific QTL allele effects from genetic background epistasis using admixed individuals in GWAS: An application to maize flowering. PLoS Genet. 16, e1008241 (2020).

72. T. C. Jaramillo, et al., Genetic background effects in Neuroligin-3 mutant mice: Minimal behavioral abnormalities on C57 background. Autism Res. 11, 234-244 (2018).

73. S. K. Remold, R. E. Lenski, Pervasive joint influence of epistasis and plasticity on mutational effects in Escherichia coli. Nat. Genet. 36, 423-
426 (2004).

74. Z. Safari, et al., Murine Genetic Background Overcomes Gut Microbiota Changes to Explain Metabolic Response to High-Fat Diet. Nutrients 12, 287 (2020).

75. C. S. Goodman, B. C. Coughlin, The evolution of evo-devo biology. Proc. Natl. Acad. Sci. 97, 44244425 (2000).

76. Sean B. Carroll, Endless Forms Most Beautiful: The New Science of Evo Devo and the Making of the Animal Kingdom (W.W. Norton \& Company, 2005).

77. J. R. True, E. S. Haag, Developmental system drift and flexibility in evolutionary trajectories. Evol. Dev. 3, 109-119 (2001).

78. M. Pavlicev, G. P. Wagner, A model of developmental evolution: Selection, pleiotropy and compensation. Trends Ecol. Evol. 27, 316-322 (2012).

79. X. Wang, R. J. Sommer, Antagonism of LIN17/frizzled and LIN-18/RyK in nematode vulva induction reveals evolutionary alterations in core developmental pathways. PLOS Biol. 9 (2011).

80. A. D. Chipman, D. H. Erwin, The evolution of arthropod body plans: Integrating phylogeny, fossils, and development-an introduction to the symposium. Integr. Comp. Biol. 57, 450-454 (2017).

81. C. K. Ewe, Y. N. Torres Cleuren, J. H. Rothman, Evolution and Developmental System Drift in the Endoderm Gene Regulatory Network of Caenorhabditis and Other Nematodes. Front. Cell Dev. Biol. 8, 1-9 (2020).

82. J. A. Arias Del Angel, A. E. Escalante, L. P. Martínez-Castilla, M. Benítez, An Evo-Devo Perspective on Multicellular Development of Myxobacteria. J. Exp. Zool. Part B Mol. Dev. Evol. 328, 165-178 (2017).

83. S. Huntley, et al., Comparative genomic analysis of fruiting body formation in Myxococcales. Mol. Biol. Evol. 28, 1083-1097 (2011).

84. Y. T. N. Yu, X. Yuan, G. J. Velicer, Adaptive evolution of an sRNA that controls Myxococcus development. Science 328, 993 (2010).

85. G. J. Velicer, H. Mendes-soares, S. Wielgoss, "Whence comes Social Diversity? Ecological and Evolutionary Analysis of the Myxobacteria" in Myxobacteria: Genomics, Cellular and Molecular Biology, Z. Yang, P. I. Higgs, Eds. (Caister Academic Press, U.K., 2014), pp. 1-28.

86. J. Hou, G. Tan, G. R. Fink, B. J. Andrews, C. Boone, Complex modifier landscape underlying genetic background effects. Proc. Natl. Acad. Sci. U. S. A. 116, 5045-5054 (2019).

87. S. V. Kadam, S. Wegener-Feldbrügge, L. Søgaard-Andersen, G. J. Velicer, Novel transcriptome patterns accompany evolutionary restoration of defective social development in the bacterium Myxococcus xanthus. Mol. Biol. Evol. 25, 1274-1281 (2008). 
88. S. A. Kraemer, G. J. Velicer, Social complementation and growth advantages promote socially defective bacterial isolates. Proc. $R$. Soc. B 281, 20140036 (2014).

89. A. Dey, et al., Sibling rivalry in Myxococcus xanthus is mediated by kin recognition and a polyploid prophage. J. Bacteriol. 198, 994-1004 (2016).

90. K. Kashefi, P. L. Hartzell, Genetic suppression and phenotypic masking of a Myxococcus xanthus frzF- defect. Mol. Microbiol. 15, 483-494 (1995).

91. P. C. Zee, et al., A shift from magnitude to sign epistasis during adaptive evolution of a bacterial social trait. Evolution 68, 2701-2708 (2014).

92. A. P. Bretscher, D. Kaiser, Nutrition of Myxococcus xanthus, a Fuiting Myxobacterium. J. Bacteriol. 133, 763-768 (1978).

93. H. Wickham, ggplot2: Elegant Graphics for Data Analysis (Springer-Verlag New York, 2016).

94. F. Sievers, et al., Fast, scalable generation of high-quality protein multiple sequence alignments using Clustal Omega. Mol. Syst. Biol. 7 (2011).

95. S. Guindon, et al., New algorithms and methods to estimate maximum-likelihood phylogenies: Assessing the performance of PhyML 3.0. Syst. Biol. 59, 307-321 (2010).

96. T. Junier, E. M. Zdobnov, The Newick utilities: high-throughput phylogenetic tree processing in the UNIX shell. Bioinformatics 26, 1669-1670 (2010).

97. E. Afgan, et al., The Galaxy platform for accessible, reproducible and collaborative biomedical analyses: 2018 update. Nucleic Acids Res. 46, W537-W544 (2018). 